\title{
Dificuldades em Avaliação Psicológica Segundo Psicólogos Brasileiros
}

\author{
Andressa Moreira Hazboun \\ João Carlos Alchieri \\ Universidade Federal do Rio Grande do Norte \\ Natal, RN, Brasil
}

\begin{abstract}
RESUMO
A avaliação psicológica (AP) se insere de forma relevante em diversos contextos de diagnóstico e intervenção, sendo cada vez mais reconhecida sua importância e necessidade. Todavia, problemas referentes à qualidade da formação, dos instrumentais utilizados e da atuação do psicólogo, preocupam profissionais e sociedade, refletindo em questões éticas. Esforços por parte de grupos e instituições têm buscado aprimorar e desenvolver a AP. Objetivando investigar as dificuldades enfrentadas no exercício profissional da AP, foi realizado um levantamento pela internet com 644 psicólogos brasileiros. Os resultados indicaram que psicólogos que atuam em contextos mais tradicionais, como clínico, escolar, do trabalho e profissional, percebem menos dificuldades em relação a profissionais dos contextos social, forense, trânsito e saúde. As principais dificuldades concernem principalmente a problemas para lidar com clientes, equipe e condições tempo e espaço.
\end{abstract}

Palavras-chave: Avaliação psicológica. Identidade profissional. Questionário online. Atuação do psicólogo.

\section{ABSTRACT}

\section{Difficulties in Psychological Evaluation According to Brazilian Professionals}

Psychological assessment (AP) is inserted relevantly in many diagnostic and intervention contexts, increasing its recognition as an important and necessary practice. However, problems concerning quality of training, use of instruments and psychologist performance, worry professionals and society, reflecting on ethical issues. Efforts by groups and institutions have sought to improve and develop AP. Aiming to investigate the difficulties encountered in the practice of AP, an online survey was conducted with 644 Brazilian psychologists. The results suggest that psychologists who work in traditional contexts, such as clinical, school, occupational and professional, perceive fewer difficulties comparing to professionals in the social, forensic, traffic and health contexts. The main difficulties are related to problems in dealing with customers, staff and conditions of time and space.

Keywords: Psychological assessment. Professional identity. Online questionnaire. Psychologist performance.

\section{RESUMEN}

\section{Dificultades en la Evaluación Psicológica Según los Profesionales Brasileños}

La evaluación psicológica (AP) se inserta relevantemente en diferentes contextos de diagnóstico e intervención y su importancia es cada vez más reconocida. Sin embargo, los problemas en la calidad de formación, instrumentos utilizados y actuación del psicólogo, preocupan los profesionales y la sociedad, incidiendo en cuestiones éticas. Los esfuerzos de grupos e instituciones han tratado de mejorar y desarrollar la AP. Con el objetivo de investigar las dificultades encontradas en la práctica de la AP, se realizó una pesquisa a través de Internet con 644 psicólogos brasileños. Los resultados indicaron que los psicólogos provenientes de contextos más tradicionales como clínica, escolar, laboral y profesional, perciben menos dificultades que los profesionales en el ámbito social, forense, de transito o de salud. Las principales dificultades se refieren a problemas en el trato con los clientes, el equipo y las condiciones del tiempo y del espacio.

Palabras clave: Evaluación psicológica. Identidad profesional. Cuestionario en línea. Actuación del psicólogo. 


\section{INTRODUÇÃO}

O cenário da avaliação psicológica (AP) tem evidenciado um largo crescimento nos últimos anos na produção científica (Barroso, 2010; Chiodi e Weschler, 2008; Joly, Berberian, Andrade e Teixeira, 2010; Souza-Filho, Belo e Gouveia, 2006; Joly, Silva, Nunes e Souza, 2007; Suehiro, 2009) e, também, protagonizado discussões para além das universidades. A presença da AP em concursos públicos, por exemplo, colocou em pauta interesses de cidadãos, psicólogos e instituições promotoras dos concursos, resultando na necessidade de formas de regulação que garantissem os direitos e deveres de todas as partes (e.g. Decreto $\mathrm{n}^{\mathrm{o}} 7.308$ de 2010). Da mesma forma, a avaliação em situações de contratação de funcionários, concessão de porte de armas ou Carteira Nacional de Habilitação (CNH), entre outros, ao mesmo tempo em que amplia a divulgação dessa prática, aumenta a exigência de fundamentação e comprovação da sua qualidade perante usuários dos serviços, instituições e profissionais de outras áreas, cuja interação com a Psicologia é cada vez mais constante (Löhr, 2011).

O Conselho Federal de Psicologia (CFP) tem incentivado o desenvolvimento da $\mathrm{AP}$, principalmente através de diversas normas reguladoras, documentos e eventos, chegando inclusive a instituir 2011 como o Ano da Avaliação Psicológica (http://anotematico. cfp.org.br/2011/). Ao lado disso, destaca-se a atuação de laboratórios e grupos de pesquisa, como o Instituto Brasileiro de Avaliação Psicológica, a Sociedade Brasileira de Rorschach e outras Técnicas Projetivas, o Laboratório de Avaliação Psicológica e Educacional da Universidade de São Paulo (USP), o Laboratório de Pesquisa em Avaliação e Medida da Universidade de Brasília (UNB), o Laboratório de Avaliação e Medidas Psicológicas da Pontifícia Universidade Católica Campinas, a Base Normativa do Comportamento Social da Universidade Federal da Paraíba, o Laboratório de Mensuração da Universidade Federal do Rio Grande do Sul, o Laboratório de Psicoterapia e Psicodiagnóstico da UNB, o Laboratório de Avaliação Psicológica da Universidade Federal do Amazonas e o Laboratório Interdepartamental de Técnicas de Exame Psicológicas do Instituto de Psicologia da USP.

Essa retomada da AP (Custódio, 2006) não a salvaguarda de críticas. Os problemas são perceptíveis, principalmente, quanto a deficiências na capacitação e treinamento oferecidos pelas universidades para a prática de AP (Noronha, Rueda, Barros e Raad, 2009; Oliveira, Noronha e Dantas, 2006; Paula, Pereira e Nascimento, 2007), uso inadequado de instrumentos psicológicos (Baumgartl, Pagano e Lacerda, 2010;
Padilha, Noronha e Fagan, 2007; Reppold, 2011; Vendramini e Lopes, 2008) e ausência de qualidades psicométricas, fundamentação científica ou atualização de alguns instrumentos (Freitas e Noronha, 2006; Paula et al., 2007). Com isso, a avaliação psicológica constitui o campo da Psicologia que abarca a maioria dos processos éticos existentes (Tavares, 2010).

Diante dos avanços, e para que a AP continue nesse intuito de desenvolvimento, é preciso definir quais os próximos passos. Nesse sentido, os levantamentos constituem métodos de pesquisa que auxiliam no delineamento de políticas que possibilitam aperfeiçoar o processo de formação para além dos limites impostos pelo mercado de trabalho. (Bastos, Gondim e BorgesAndrade, 2010). Assim, realizou-se um levantamento com psicólogos brasileiros no qual, dentre os aspectos abordados, buscou-se identificar as dificuldades observadas nos diversos contextos em que se realiza AP.

\section{MÉTODO}

O levantamento foi realizado através de um questionário online, divulgado pessoal e virtualmente, com uso de uma estratégia bola-de-neve envolvendo: setores de comunicação e/ou psicólogos inseridos em entidades ou instituições ligadas à Psicologia e psicólogos cadastrados em redes sociais na internet. A pesquisa foi aprovada pelo comitê de Ética da Universidade Federal do Rio Grande do Norte sob o parecer de número 096/10. Foram analisadas as respostas da seguinte questão aberta "Na sua opinião, quais as maiores dificuldades de se trabalhar com avaliação psicológica no contexto X?" Sendo "X" o contexto de atuação indicado pelo participante dentre as opções: Clínico; Vocacional/Profissional; Saúde/Hospitalar; Social/Comunitário; Porte de Armas; Trânsito/CNH; Forense/Jurídico; Trabalho/ Organizacional; Escolar/Educacional; Outro contexto.

A opção pelo formato aberto da questão deveuse ao intento de priorizar a aspectos da compreensão e representação que o psicólogo constrói acerca das dificuldades que enfrenta e que considera importantes, como se posiciona diante dos entraves da sua área e expressa suas necessidades.

Os procedimentos de análise foram representados em termos de análises descritivas e inferencial e os resultados foram descritos em porcentagens válidas. Os dados textuais foram processados com auxílio do software Alceste (Análise Lexical por Contexto de um Conjunto de Segmentos de Texto), através do qual os corpus com as respostas dos participantes, ou Unidades de Contexto Iniciais (UCI), são divididas em Unidades de Contexto Elementar (UCE), cuja distribuição do 
vocabulário em termos de frequência e Qui-quadrado $\left(\chi^{2}\right)$ permite a criação de grupos ou classes de palavras significativas, com frequência média superior a quatro (critério lexicográfico fornecido pelo próprio software). No caso do Qui-quadrado, considera-se significativo valores acima de 3,84 (Camargo, 2005)

\section{RESULTADOS}

Dos 644 psicólogos que alegaram praticar AP, a idade situou-se entre 22 e 66 anos ( $37 \pm 10,7$ anos), sendo $12,2 \%$ até 25 anos, $42,5 \%$ de 26 a 35 anos, $20,8 \%$ de 36 a 45 anos, $18,9 \%$ de 46 a 55 anos, $5 \%$ de 56 a 65 anos e $0,6 \%$ com mais de 65 anos. A maioria (37,5\%) graduou-se após 2005, 24,4\% entre 2001 e 2005, 8,9\% entre 1996 a 2000 e também 8,9\% entre 1991 e 1995 , $7,2 \%$ entre 1986 e $1990,7,8 \%$ entre 1981 e 1985 e $5,3 \%$ até 1980 . O tempo de prática em AP de 39,8\% dos psicólogos foi de menos de 4 anos, $26,7 \%$ de 4 a 8 anos, $11,5 \%$ de 9 a 13 anos, $7,9 \%$ de 14 a 18 anos, $6,4 \%$ de 19 a 23 anos e 7,8\% com mais de 23 anos.

Quanto ao principal contexto de atuação, a grande maioria dos psicólogos (41,1\%) indicou o Clínico (C), seguida de 16,1\% no Trabalho/Organizacional (T/O), 13,8\% em Saúde/Hospitalar (S/H), 8,1\% em Escolar/ Educacional (E/E), 6,5\% no Forense/Jurídico $(\mathrm{F} / \mathrm{J})$, 5,6\% no contexto de Trânsito/CNH (T/CNH), 4,5\% no Vocacional/Profissional (V/P), 2,3\% no Social/ Comunitário (S/C), 0,9\% em Porte de Armas (PA) e $0,9 \%$ em outros contextos $(\mathrm{O})$.

No total, $54 \%$ dos profissionais alegaram não perceber dificuldades em praticar AP no contexto onde atua e $46 \%$ observaram dificuldades.

Foram analisadas diferenças na percepção de dificuldades quanto à região do Brasil e ao público atendido. Houve diferença significativa $(p=0,014)$ indicando uma associação entre a percepção de dificuldades e as regiões Norte, Nordeste e CentroOeste, bem como uma associação entre a não percepção de dificuldades e a regiões Sudeste. Quanto ao público atendido, somente o atendimento de idosos representou significância estatística $(p=0,000)$, evidenciando uma associação entre a percepção de dificuldades e o atendimento desse público.

Também se verificaram diferenças estatisticamente significativas $(p<0,001)$ na percepção de dificuldades para diferentes contextos, cujas frequências podem ser elucidadas na Tabela 1. Observa-se que os contextos forense, trânsito, saúde, social e outros, superam em percepção de dificuldades.

Embora 46\% do total de psicólogos participantes da pesquisa tenham mencionado observar dificuldades em praticar AP, 33\% foram os que relataram quais seriam essas dificuldades. Tais relatos foram analisados com auxílio do Alceste, a partir de um corpus composto por 215 Unidades de Contexto Inicial (UCI), contendo um total de 3705 palavras, cuja riqueza do vocabulário foi calculada em $95 \%$. O Alceste gerou, a partir do corpus de análise, 230 Unidades de Contexto Elementar (UCE), das quais $80 \%$ deram origem a 5 classes estáveis.

As expressões mais significativas de cada classe com os respectivos valores do $\chi^{2}$ entre parênteses estão elencados na Tabela 2, ao lado de cada classe está a porcentagem de UCEs classificadas.

A partir das formas mais significativas e da leitura das UCEs de cada classe foi referido um tema central. A primeira denominou-se "condições tempo-espaço e relacionamento com a equipe", uma vez que as dificuldades relatadas centravam-se principalmente em dar conta e uma grande demanda de AP diante de condições limitadas de tempo e espaço, bem como nas dificuldades no relacionamento com outros profissionais. Exemplos disso podem ser evidenciados nos discursos: “(...) interrupções devido à equipe multiprofissional." (S.200), "dificuldade de tempo para uma boa avaliação psicológica devido à demanda de trabalho.” (S.255), "(...) dificuldade de entrosamento enquanto equipe multiprofissional. Pressão para realização do trabalho em curto espaço de tempo (...)" (S.290), "espaço inadequado. tempo reduzido (...)" (S.324). Foram ainda relevantes nessa categoria psicólogos advindos do contexto social-comunitário $\left(\chi^{2}=6\right)$, com titulação máxima de graduação $\left(\chi^{2}=12\right)$.

A segunda classe foi designada "custo-benefício" e refere-se principalmente aos testes e convênios de saúde. Os primeiros são criticados por serem caros, carentes de qualidades psicométricas, principalmente para populações específicas e, ainda, pela ausência de especificidade suficiente pra alguns tipos de avaliação. São exemplos disso trechos como: “(...). alguns testes utilizados em países desenvolvidos não

TABELA 1

Percepção de dificuldades por contexto de atuação

\begin{tabular}{ccccccccccc}
\hline Dificuldades & $E / E$ & $P A$ & $V / P$ & $T / O$ & $C$ & $F / J$ & $T / C N H$ & $S / H$ & $S / C$ & $O$ \\
Sim & 45,1 & 33,3 & 31,0 & 38,6 & 36,3 & 76,2 & 70,6 & 62,4 & 57,1 & 100,0 \\
Não & 54,9 & 66,7 & 69,0 & 61,4 & 63,7 & 23,8 & 29,4 & 37,6 & 42,9 & \\
\hline
\end{tabular}


TABELA 2

Classes de UCEs e formas significativas

\begin{tabular}{|c|c|c|c|c|}
\hline Classe $1(25 \%)$ & Classe $2(16 \%)$ & Classe $3(38 \%)$ & Classe $4(14 \%)$ & Classe $5(7 \%)$ \\
\hline $\begin{array}{l}\text { - tempo (69) } \\
\text { - demandar (23) } \\
\text { - curto (22) } \\
\text { - corrigir (18) } \\
\text { - atendimento (18) } \\
\text { - equipe (16) } \\
\text { - trabalhar (15) } \\
\text { - vez (9) } \\
\text { - avaliações psicológicas (9) } \\
\text { - dificuldade (8) } \\
\text { - aplicar (6) } \\
\text { - maior (6) } \\
\text { - saúde (6) } \\
\text { - grande (6) } \\
\text { - realizar (6) } \\
\text { - avaliar (6) } \\
\text { - inadequado (4) }\end{array}$ & $\begin{array}{l}\text { - conselho federal de } \\
\text { psicologia (49) } \\
\text { - população (42) } \\
\text { - valor (38) } \\
\text { - brasil (38) } \\
\text { - aprovados (32) } \\
\text { - custo (27) } \\
\text { - convênios de saúde (27) } \\
\text { - alto (21) } \\
\text { - crianças (16) } \\
\text { - realidade (16) } \\
\text { - validados (7) } \\
\text { - pais (6) } \\
\text { - ausência (6) } \\
\text { - padronizados (6) } \\
\text { - conselho regional (6) } \\
\text { - teste (5) } \\
\text { - alguns (5) } \\
\text { - não (4) } \\
\text { - inadequado (4) }\end{array}$ & $\begin{array}{l}\text { - cliente (16) } \\
\text { - avaliação psicológica (16) } \\
\text { - psicólogo (12) } \\
\text { - conhecimento (12) } \\
\text { - sobre (9) } \\
\text { - objetivo (9) } \\
\text { - processo (8) } \\
\text { - sem (7) } \\
\text { - parte (7) } \\
\text { - de que (7) } \\
\text { - necessidade (7) } \\
\text { - poderia (5) } \\
\text { - momento (5) } \\
\text { - resultado (5) } \\
\text { - disponíveis (5) } \\
\text { - uma vez que (5) } \\
\text { - padronização (5) } \\
\text { - ser (4) } \\
\text { - situação (4) } \\
\text { - pesquisa (4) }\end{array}$ & $\begin{array}{l}\text { - profissional (35) } \\
\text { - adequado (30) } \\
\text { - estrutura (30) } \\
\text { - capacitação (30) } \\
\text { - falta (24) } \\
\text { - material (15) } \\
\text { - instituição (11) } \\
\text { - sigilo (9) } \\
\text { - escola (7) } \\
\text { - física (7) } \\
\text { - cursos (7) } \\
\text { - área (5) } \\
\text { - informação (5) } \\
\text { - espaço (4) } \\
\text { - atualização (4) }\end{array}$ & $\begin{array}{l}\text { - interpretação (82) } \\
\text { - baixo (72) } \\
\text { - nível de escolaridade (72) } \\
\text { - local (7) } \\
\text { - cliente (7) } \\
\text { - cultura (5) }\end{array}$ \\
\hline
\end{tabular}

foram aprovados pelo Conselho Federal de Psicologia por motivos distintos ou não foram adaptados ou publicados a nossa população.” (S.92), “(...) normas de testes defasadas. alto custo de alguns testes para a instituição publica." (S.279), "falta de testes aprovados pelo Conselho Federal de Psicologia direcionados as crianças." (S.515). Quanto aos convênios de saúde, a principal dificuldade provém da remuneração, como pode ser percebido em: "(...) baixo valor pago pelos convênios (de saúde)" (S.228), "os valores que os convênios de saúde pagam são insignificantes diante de tamanho investimento e responsabilidade." (S.504). Foram relevantes, nessa classe, psicólogos atuantes no contexto clínico $\left(\chi^{2}=12\right)$.

Denominou-se "clientes e instrumentos" a terceira classes. Psicólogos do contexto Trânsito/CNH $\left(\chi^{2}=7\right)$ tiveram mais peso para configuração desta classe. As preocupações com os clientes concernem tanto às expectativas quanto às atitudes não colaborativas, as quais, muitas vezes, são reforçadas pela fragilidade dos instrumentos, como no exemplo: "O fato de os instrumentos, em geral, envolverem autopercepção, podem sofrer manipulações ou distorções (...)" (S.342), "O pouco conhecimento dos clientes referente ao processo de avaliação psicológica." (S.357), "falta de seriedade e empenho dos candidatos quanto à avaliação psicológica. (...) por outro lado, muitos candidatos ficam ansiosos no processo (...)" (S.70). Os instrumentos são também questionados quanto à qualidade, a ausência de pesquisas e padronização, mas há também críticas quanto aos próprios profissionais no uso desses instrumentos: "os próprios psicólogos banalizam a avaliação psicológica, utilizando instrumentos que não são válidos ou tirando cópias dos mesmos." (S.553), "as maiores dificuldades pairam em torno da reflexão crítica do psicólogo sobre a falta de especialistas e a necessidade constante de atualizações dos instrumentos e técnicas de avaliação psicológica para avaliar os fenômenos e processos psicológicos." (S.420).

A quarta classe foi nomeada "materiais, estrutura e capacitação" teve maior participação do contexto Saúde/ Hospitalar $\left(\chi^{2}=4\right)$. Foi recorrente nesta classe a menção de dificuldades relacionadas a materiais, adequada estrutura física e capacitação, principalmente, em se tratando de instituições. Discursos característicos são: "as instituições não adquirem o material necessário, gerando um ônus para os profissionais (...)" (S.598), "acredito que faltam ofertas de cursos para capacitação profissional (...)" (S.568), "A instituição escolar não possui estrutura favorável, pois tem barulho $e$ interrupções." (S.341), "falta de capacitação dos profissionais." (S.413), "falta de estrutura fornecida pela prefeitura (...) falta de incentivo para capacitação, aprimoramento e estudo do profissional." "falta de material. Material caro." (S.74).

À última classe foi conferido o título "adequação cultural", em referência a dificuldade de lidar com o baixo nível de escolaridade de diversos clientes e instrumentos insuficientemente qualificados para certas 
realidades sócio-culturais. Muito embora haja queixas como dificuldades de tempo, espaço e capacitação (já mencionadas em outras classes) há maior ênfase com relação aos entraves socioculturais na prática da AP. Exemplos de UCEs significativas, de acordo com o Alceste, são: "O baixo nível de escolaridade da maioria dos clientes adultos, analfabetos ou com ensino fundamental incompleto, o que dificulta a interpretação de muitos testes." (S.197), "aliar a bateria de testes à idade, nivel de escolaridade e cargo. Os manuais não dão o suporte necessário a interpretação e avaliação." (S.38), “(...) dificuldade dos clientes em compreender as questões (...)" (S.42), "difícil interpretação de alguns testes. Falta tabela para minha região, tendo sempre que procurar a que mais se aproxima do tipo de cultura ou local (...)." (S.34). Nessa classe, foram relevantes respondentes do CRP-14 $\left(\chi^{2}=9\right)$, com 5 a 8 anos de prática $\left(\chi^{2}=7\right)$, atuando no contexto do trabalho/ organizacional $\left(\chi^{2}=5\right)$ e especialistas $\left(\chi^{2}=4\right)$.

\section{DISCUSSÃO}

Muitos dos problemas em AP costumam ser atribuídos a deficiências na formação e/ou à ausência ou inadequabilidade de instrumentais que poderiam indicar, junto aos problemas éticos decorrentes, dificuldades na adequada execução dessa atividade. Não obstante, a maioria dos participantes relatou não perceber dificuldades. Esse é um dado que chama atenção, principalmente em se tratando de psicólogos recém-formados, deparando-se com as contingências do mercado de trabalho, ou com um conhecimento tácito incipiente ante o pouco tempo de experiência. Por outro lado, pode ser justamente por estarem iniciando o exercício profissional que muitos psicólogos tenham tido poucas oportunidades de vivenciar a prática, ou mesmo, de se deparar com dificuldades relevantes.

A menor percepção de dificuldades na região Sudeste pode estar relacionada a tradicional hegemonia dessa na concentração de psicólogos, cursos de graduação e pós-graduação, eventos e produção em Psicologia (Bastos et al., 2010), podendo a Psicologia encontrar-se mais consolidada e difundida nessa região. Já nas regiões onde a Psicologia ainda busca espaço, faz sentido uma maior percepção de problemas citados, como expectativas e atitudes negativas de clientes, demanda excessiva, estrutura deficiente, dificuldades no relacionamento com a equipe e de acesso a materiais, entre outros. Além disso, queixas como o baixo nível de escolaridade de clientes e instrumentos insuficientemente qualificados para certas realidades socioculturais possivelmente são menos pertinentes ao Sudeste onde se realizam a maioria das pesquisas e o índice de escolaridade da população é maior, se comparado, por exemplo, à região Nordeste que detém o maior número de analfabetos em relação às outras regiões do país (Instituto Brasileiro de Geografia e Estatística - IBGE, 2010). Outro ponto que é importante mencionar refere-se à percepção de dificuldades associada ao atendimento de idosos. Esse dado pode indicar lacunas na capacitação profissional, considerando um provável aumento da demanda ante o crescimento expressivo dessa parcela da população no Brasil (Organização Mundial de Saúde - OMS, 2005) cujo ritmo não é acompanhando pelos os estudos voltados para a terceira idade.

Também são apontadas disparidades na observação de dificuldades por psicólogos inseridos em diferentes contextos, reforçando a necessidade de práticas específicas de AP, para além do modelo clínico (Araújo, 2007). A percepção de dificuldades por um grupo menor no contexto clínico pode se dever a larga tradição que a AP tem nesse contexto, para o qual se volta a maioria dos estudos, instrumentos e procedimentos, reforçada pela própria tradição clínica na formação em Psicologia (Araújo, 2007; Joly et al., 2010). Ainda assim, os psicólogos clínicos relataram dificuldades relevantes para constituição da classe "custo-benefício", provavelmente, por ser característica desse contexto a vinculação a convênios de saúde.

Os contextos escolar, trabalho e profissional, também possuem uma forte tradição na $\mathrm{AP}$, diferentemente do contexto social, por exemplo, onde mais dificuldades foram percebidas, principalmente com relação às condições de tempo, espaço e relacionamento com a equipe. Tendo em vista que só recentemente o psicólogo inseriu-se nos contextos de assistência social, com a Política Nacional de Assistência Social (PNAS/2004) e o Sistema Único da Assistência Social (SUAS, 2005), pode-se supor que há, justificadamente, um processo de consolidação da atuação nessa área, inclusive no que tange às práticas de AP. Além disso, saber trabalhar em equipes inter e multidisciplinares é praticamente uma exigência da atualidade (Löhr, 2011) para qual os psicólogos, talvez, não estejam sendo bem preparados. Já as dificuldades com relação ao tempo podem estar relacionadas a peculiaridades dos contextos que tiveram uma expressiva identificação de dificuldades (forense, trânsito, saúde e social). No caso da AP para $\mathrm{CNH}$, por exemplo, o crescimento da demanda ao lado da pressão pela rapidez dos resultados, leva o psicólogo a necessitar de estratégias para dar conta do trabalho (Silva e Alchieri, 2010). Também o contexto forense é marcado por entraves relacionados ao tempo, dada à urgência que geralmente envolve as decisões processuais (Costa, Penso e Sudbrack, 2009). 
$\mathrm{Na}$ análise das dificuldades mencionadas pelos participantes, a classe com maior representatividade foi denominada "clientes e instrumentos", relacionada a problemas com a qualidade dos instrumentos utilizados e as expectativas dos clientes. É interessante notar que a significância de profissionais do contexto de Trânsito/ $\mathrm{CNH}$ para configuração dessa classe pode estar relacionada ao que SantAnna e Bastos (2011) apontam como a "desvalorização dos instrumentos associada à desvalorização do próprio profissional" (p. 118) que, dentre outras limitações, não possui autonomia sequer para escolher os instrumentos que são determinados pelos Departamentos Estaduais de Trânsito.

Foram abordadas também dificuldades relacionadas à adequada estrutura física e capacitação, principalmente, em se tratando de instituições e com relevância do contexto Saúde/Hospitalar. Em menor grau, citaram-se as dificuldades de lidar com o baixo nível de escolaridade de diversos clientes e instrumentos insuficientemente qualificados para certas realidades socioculturais. Diante de uma realidade brasileira em que muitos não têm acesso à educação de qualidade, Alchieri (2010) aponta a necessidade de se objetivar estudos e investigações às demandas sociais. Quanto à estrutura física das instituições é importante salientar que, caso estas não estejam adequadas a uma prestação de qualidade dos serviços psicológicos, considera-se uma infração do Art. $1^{\circ}$ do código de Ética (2005). No compromisso ético de psicólogo, perpassa também a sua capacitação. Todavia, o trabalho em instituições parece um grande desafio diante de uma formação tradicionalmente clínica que reflete nas aplicações da AP e seus procedimentos (Araújo, 2007; Joly et al., 2010) que necessitam ser contextualizados.

\section{CONSIDERAÇÕES FINAIS}

Muitos dos problemas relacionados à AP costumam ser atribuídos a deficiências na formação ou à escassez/ inadequabilidade de instrumentais, que resultam em questões éticas e, poderiam indicar dificuldades na adequada execução dessa atividade. Não obstante, a maioria dos participantes relatou não perceber dificuldades. Esse dado chama atenção, principalmente, em se tratando de psicólogos recém-formados, ou seja, deparando-se com as contingências do mercado de trabalho e, possivelmente, com um conhecimento tácito incipiente. Por outro lado, pode ser justamente por estar iniciando o exercício profissional que tais psicólogos tenham tido poucas oportunidades de vivenciar dificuldades relevantes.

Não obstante as limitações desse estudo, cabe questionar a postura dos profissionais ante o seu exercício, bem como o nível de reflexão, pois se não há dificuldades a serem enfrentadas, não haverá conhecimento a ser buscado. É claro que os profissionais podem, de fato, estar realizando seu exercício sem maiores entraves. Porém, diante de um quadro preocupante de processos éticos e dado o próprio desafio que é trabalhar com AP, será que o psicólogo está julgando adequadamente sua atividade? Se este profissional não foi avaliado adequadamente durante sua formação e, se o público a que presta serviços pouco entende para criticar o seu trabalho, há poucas chances de que este perceba dificuldades no seu labor.

\section{REFERÊNCIAS}

Alchieri, J. C. (2010). Análise dos dados demográficos das normas brasileiras de instrumentos psicológicos empregados na avaliação da personalidade. Revista Psicologia e Saúde, 2(1), 56-63.

Araújo, M. F. (2007) Estratégias de diagnóstico e avaliação Psicológica. Psicologia: Teoria e Prática, 9(2),126-141.

Barroso, S. M. (2010). Avaliação psicológica: análise das publicações disponíveis na Scielo e BVS-Psi. Fractal: Revista de Psicologia, 22(1), 141-154.

Bastos, A. V. B., Gondim, S. M. G., \& Borges-Andrade, J. E. (2010). O psicólogo brasileiro: Sua atuação e formação profissional. O que mudou nas últimas décadas? In O. H. Yamamoto, \& A. L. F. Costa (Orgs.), Escritos sobre a profissão de psicólogo no Brasil (pp. 257-271). Natal: EDUFRN.

Baumgartl, V. O., Pagano, A. P., \& Lacerda, J. (2010). A utilização de testes psicológicos em organizações de Minas Gerais. Arquivos Brasileiros de Psicologia, 62(2), 178-186.

Camargo, B. V. (2005). Alceste: um programa informático de análise quantitativa de dados textuais. In A. S. P. Moreira (Org.). Perspectivas teórico-metodológicas em representações sociais (pp. 511-539). João Pessoa: UFPB/Editora Universitária.

Chiodi, M. G., \& Weschler, S. M. (2008). Avaliação psicológica: contribuições brasileiras. Boletim Academia Paulista de Psicologia, (02/08), 197-210.

Costa, L. F., Penso, M. A., Sudbrack, V. N. L. (2009). As competências da Psicologia Jurídica na avaliação psicossocial de famílias em conflito. Psicologia \& Sociedade, 21(2), 223-241.

Custódio, E. M. (2006). Avaliação psicológica ensino e pesquisa na década de sessenta. Boletim Academia Paulista de Psicologia, 2(7), 49-66.

Decreto $n^{\circ} 7.308,\left(2010,22\right.$ de setembro). Altera o Decreto $n^{\circ} 6.944$, de 21 de agosto de 2009, no tocante à realização de avaliações psicológicas em concurso público. Brasília, DF: Presidência da República.

Freitas, F. A., \& Noronha, A. P. P. (2005). Clínica-escola: levantamento de instrumentos utilizados no processo psicodiagnóstico. Psicologia Escolar e Educacional, 9(1), 87-93.

Instituto Brasileiro de Geografia e Estatística - IBGE. (2010). Percentual de pessoas de 10 anos ou mais de idade alfabetizadas, segundo as grandes regiões - 2010. Sinopse do Censo Demográfico 2010. Recuperado de http://www.censo2010.ibge.gov.br/ sinopse/index.php?dados $=\mathrm{P} 6 \& u \mathrm{f}=00$

Joly, M. C. A. R., Berberian, A. A., Andrade R. G., \& Teixeira, T. C. (2010). Análise de teses e dissertações em avaliação psicológica disponíveis na BVS-PSI Brasil. Psicologia Ciência e Profissão, 30(1), 174-187. 
Joly, M. C. R. A., Silva, M. C. R., Nunes, M. F. O., \& Souza, M. S. (2007). Análise da produção científica em painéis dos congressos brasileiros de avaliação psicológica. Avaliação Psicológica, 6(2), 239-252.

Löhr, S. S. (2011). Avaliação psicológica na formação do profissional da Psicologia, algumas reflexões. In Grupo de Trabalho do Ano Temático da Avaliação Psicológica (Org.). Ano da Avaliação Psicológica - Textos geradores (pp. 143-149). Brasília: Conselho Federal de Psicologia.

Ministério do Desenvolvimento Social e Combate à Fome. (2004). Politica Nacional de Assistência Social-PNAS. Brasília.

Ministério do Desenvolvimento Social e Combate à Fome. (2007). SUAS: Sistema Único de Assistência Social. Brasília.

Noronha, A. P. P., Rueda, F. J. M., Barros, M. V. C., \& Raad, A. J. (2009). Estudo transversal com estudantes de psicologia sobre conceitos de avaliação psicológica. Psicologia Argumento, 27(56), 77-86.

Oliveira, K. L., Noronha, A. P. P., Dantas, M. A. (2006). Instrumentos psicológicos estudo comparativo entre estudantes e profissionais cognitivo-comportamentais. Estudos de Psicologia Campinas, 23(4), 359-367.

Organização Mundial de Saúde (2005). Envelhecimento ativo: uma política de saúde. Brasília: Organização Pan-Americana da Saúde.

Padilha, S., Noronha, A. P. P., \& Fagan, C. Z. (2007). Instrumentos de avaliação psicológica: uso e parecer de psicólogos. Avaliação Psicológica, 6(1), 69-76.

Paula, A. V., Pereira, A. S., \& Nascimento, E. (2007). Opinião de alunos de psicologia sobre o ensino em avaliação psicológica. PsicoUSF, 12(1), 33-43.

Resolução CFP nº 010/2005 (2005, 21 de julho). Aprova o Código de Ética Profissional do Psicólogo. Brasília, DF: Conselho Federal de Psicologia.

Reppold, C. T. (2011). Qualificação da avaliação psicológica: critérios de reconhecimento e validação a partir dos Direitos Humanos. In Grupo de Trabalho do Ano Temático da Avaliação
Psicológica (Org.). Ano da Avaliação Psicológica - Textos geradores (pp. 21-28). Brasília: Conselho Federal de Psicologia.

SantAnna, J., \& Bastos, P. (2011). Relações institucionais: a avaliação psicológica no contexto trânsito. In Grupo de Trabalho do Ano Temático da Avaliação Psicológica (Org.), Ano da Avaliação Psicológica - Textos geradores (pp. 115-119). Brasília: Conselho Federal de Psicologia.

Silva, F. H. V. C. \& Alchieri, J. C. (2010). Validade preditiva de instrumentos psicológicos usados na avaliação psicológica de condutores. Psicologia: Teoria e Pesquisa, 6(4), 695-706.

Souza-Filho, M. L. de, Belo, R., \& Gouveia, V. V. (2006) Testes psicológicos: análise da produção científica brasileira no período 2000-2004. Psicologia Ciência e Profissão, 26(3), 478-489.

Tavares, M. (2010). Da ordem social da regulamentação da avaliação psicológica e do uso dos testes. In Conselho Federal de Psicologia (Org.). Avaliação Psicológica. Diretrizes na regulamentação da profissão (pp. 31-56). Brasília: Conselho Federal de Psicologia.

Vendramini, C. M. M., \& Lopes, F. L. (2008). Leitura de manuais de testes psicológicos por estudantes e profissionais de psicologia. Avaliação Psicológica, 7(1), 93-105.

\section{Autores:}

Andressa Moreira Hazboun - Mestre em Psicologia. Universidade Federal do Rio Grande do Norte.

João Carlos Alchieri - Doutor em Psicologia. Universidade Federal do Rio Grande do Norte.

\section{Endereço para correspondência:}

Andressa Moreira Hazboun

Universidade Federal do Rio Grande do Norte

Rua Maxaranguape, 550/2003 - Tirol

CEP: 59020160 Natal, RN

E-mail: dxahaz@gmail.com

Recebido em: 14.02.2013.

Aceito em: 22.10.2013. 